\title{
Generalized entropies and non-linear kinetics
}

Entropy represents one of the most all-encompassing underlying concepts in science. Evolution equations and conservation laws, as well as symmetries in fundamental theories and complex system models, all follow elegantly by maximizing certain entropic functionals. In particular, in statistical physics entropy is a very powerful and efficient investigative tool, both in equilibrium statistical mechanics and in kinetic theory.

The importance of entropy, in both its ordinary and generalized forms, is demonstrated in the twelve original papers collected in this special issue of the European Physical Journal B. The first five papers deal with the theoretical and mathematical aspects of some generalized entropies and the combinatorial basis of Boltzmann entropy. The subsequent three papers consider non-linear kinetics from a general perspective, while the final four papers examine kinetic and stochastic models with applications in specific complex physical systems. We hope that these articles will provide interesting insight into recent developments in modern Statistical Physics.

The papers in this issue have been selected and invited for submission based on presentations at the conference SigmaPhi2008 held in Crete last year. They represent regular research articles, which underwent the standard rigorous editorial process of the EPJB. For the considerable effort during the review process, we wish to warmly thank the Referees, along with all the other people that contributed in different ways to the preparation of this issue.

The Guest Editors

Giorgio Kaniadakis and Antonio Scarfone Politecnico di Torino, Italy 